This document is published in:

"IEEE Transactions on Industrial Informatics, vol. 10, n. 1, February 2014, pp.

680-688. DOI: http://dx.doi.org/10.1109/TII.2013.2246173"

(C) 2013 IEEE. Personal use of this material is permitted. Permission from IEEE must be obtained for all other uses, in any current or future media, including reprinting/republishing this material for advertising or promotional purposes, creating new collective works, for resale or redistribution to servers or lists, or reuse of any copyrighted component of this work in other works.

This work was supported by the Spanish Government through the project call "A new approach to social robotics (AROS) of MICNN (Ministry of Science and Innovation). Paper no. TII-12-0561, and supported by the CAM Project S2009/DPI1559/ROBOCITY2030 II, developed by the research team RoboticsLab at the University Carlos III of Madrid. 


\title{
Signage system for the navigation of autonomous robots in indoor environments
}

\author{
Ana Corrales, Maria Malfaz, and Miguel A. Salichs
}

\begin{abstract}
In many occasions people need to go to certain places without having any prior knowledge about the environment. This situation may occur when the place is visited for the first time, or even when there is not any available map to situate us. In those cases, the signs of the environment are essential for achieving the goal. The same situation may happen for an autonomous robot. This kind of robots must be capable of solving this problem in a natural way. In order to do that, they must use the resources present in their environment. This paper presents a RFID-based signage system, which has been developed to guide and give important information to an autonomous robot. The system has been implemented in a real indoor environment and it has been successfully proved in the autonomous and social robot Maggie. At the end of the paper some experimental results, carried out inside our university building, are presented.
\end{abstract}

Index Terms-Signage system, smart homes, building automation, RFID, wayfinding, navigation, autonomous robot, social robot.

\section{INTRODUCTION}

$\mathbf{O}$ $\mathrm{NE}$ of the main research goals of social robotics is the incorporation of robots in environments surrounded by people, for example, at home, hospitals, museums, offices, factories, etc. In those places, the autonomy of a social robot plays a key role and navigation is one of its needed skills.

Frequently, people need to go to a particular place without having any prior knowledge of the environment (it is our first time in that place, or we do not have a map). The human process of orientation, searching, and following a way toward a specific goal, is a cognitive process extensively studied in fields such as psychology [1][2], anthropology [3], and geography [4]. This cognitive process is known by the term "Wayfinding".

There are many daily situations which motivate us to activate this process. For example, when we are at an unknown airport and we need to go to a certain boarding gate. In this case, we need to find the signs around, and follow their indications to reach our goal. As already said, with no previous knowledge of the path to follow, the information given by the environment is essential to find the right way. As humans must deal with this kind of situations, an autonomous social robot, which is intended to move in the same environment as humans, must be also able to solve this problem. That is, it must be capable of searching for signs and, using their information, finding its goal.

The main objective of this work is to design a signage system for autonomous robots. This system will facilitate the

The authors are with the Robotics Lab of Carlos III University of Madrid, Leganés, Spain.

(e-mail: [acorrale, mmalfaz, salichs]@ing.uc3m.es ) navigation of the robot in different environments, such as, homes and buildings, in situations where it must fulfill a navigation mission and it has no previous knowledge about those places. It is expected that the robot uses the signage system in a natural way as humans do during the wayfinding process.

The majority of the smart homes applications are oriented to make the environment more comfortable for people [5]. However, our objective is to incorporate Radio Frequency Identification (RFID) technology in those environments to facilitate the navigation of autonomous robots, as future human companions. In order to implement the proposed signage system, it is necessary to slightly modify the environment by adding the signals. Therefore, the robot has access to the needed information to guide itself in the environment and to carry out its navigation task successfully.

Moreover, in this paper, a navigation algorithm is also presented. This algorithm specifies the searching process of the signals and their interpretation. Then, the skills of the robot are coordinated in order to arrive at the specified destination.

The proposed signage system has been implemented in a real environment, our university department, an indoor environment with a main corridor and several offices. Moreover, the navigation algorithm has also been implemented in a real social and autonomous robot named Maggie. At the end of the paper, some experimental results are shown.

This paper is organized as follows: Section II shows some related works which use signals as support for navigation tasks, mainly those using RFID technology. Next, the design of the signage system is explained in Section III and its implementation in a real robot is described in Section IV. Section V presents the developed navigation algorithm which uses the information stored in the signals of the environment. Later, some experiments, carried out with the social robot Maggie in a real environment, are shown in Section VI. Finally, in Section VII, the main conclusions and some future works are presented.

\section{RELATED WORK}

The main goal of a signage system is to guide and inform users in order to arrive at their destinations. The design of a sign system depends on the needs of the users and in this case, the users are autonomous robots. These robots must be able to easily access to the information given by the signals in order to successfully navigate without disturbing others (people or other robots).

There are some related works which use signals in the environment to support navigation. Some of them use marks 
or reference points to localize the robot in the environment, and many of them use artificial vision techniques to detect them. In [6], the authors present a visual perception and recognizer system of natural marks (designed for humans) in indoor environments (for example, the identification tags in the offices's doors). In [7] a topological navigation system for mobile robots is presented. This system is also applied in indoor environments and they use an artificial mark called $M R$ (Mobile Robot) code. Moreover, in this approach a topological map is previously needed. The main disadvantages of this kind of solutions is the small amount of information stored and their dependency on the lighting conditions.

Recently, the RFID technology has been presented as a new tool for facilitating and improving the acquisition of the environment information [8] [9] [10] [11]. Then, they favor the people and objects identification process. There are some works which use RFID tags as artificial marks in the environment. Most of them use those marks to solve the geometric localization problem by storing the geometric position of the tag in relation to a map of the environment [12] [13] [14].

On the other hand, in relation to the development of supporting systems for robot navigation using RFID technology there are not so many works. Many of the applications found are oriented to give support to disabled or elderly people. In the University of Utah [15], an assistant robot for blind people has been implemented. This robot uses RFID tags to guide the people, in indoor environments, by giving information of the current place and guiding them to the desired destination. The main problem of this algorithm is the possibility of getting lost or failure in recognizing the tags. There is not an estimation method for the lost nodes, as it happens when there are moving obstacles in the environment. The developed algorithm as been also implemented in other applications: to help blind people while shopping in a supermarket [16]; and as a support system for elderly people using a wheeled smart walking frame, named iWalker [17]. The main disadvantage of those prototypes is that the routes must be pre-coded and they also require a map of the environment.

As previously said, in this work a signage system is presented. Its goal is to serve as a robot navigation support when the robot is at an unknown place with no previous information. The robot must be able to guide itself and to arrive at its destination using only the information given by the signals. In order to do this, it is required to include signals in the environment. Those signals must provide the robot with the needed information and, at the same time, they cannot affect the navigation of people, nor have a negative impact over the environment. For these reasons, the RFID technology has been selected to design the signage system presented in this paper.

\section{Signage System}

Signage can be defined as the set of elements situated in the environment that, through sensorial indications, guides and help users to navigate in an area or building. It serves as support for the orientation and helps the accessibility to the required services, which gives security during displacements and actions.
In this context, the signal can be defined as a type of sign situated in the environment to identify an element or place, or to give an advise or indication. Moreover, the signal serves to guide the user in the environment.

The proposed signals have been designed considering the following:

- The information of the signal must facilitate the required knowledge through a clear message.

- The information must not be confusing or excessive.

- The signals must be easily accessible. In the case of robots, this will depend on the sensor which receives the information, in this case, it will depend on the features of the RFID system.

- The signals must not affect the environmental harmony, that is, they must not affect the environment aesthetically, nor make the navigation difficult.

The information used from the environment will depend on: the sensory system of the robot, the structure of the environment, and the information required by the robot to arrive at its destination. In the case that the robot does not have a previous knowledge of the environment, the information of the signals must give answers to the following questions: where I am?, How can I get there?, and where can I go to?.

1) Where I am?: one of the main problems that must be solved by an autonomous robot is its localization. Then, it is necessary to design Identifying Signals indicating the name of the place where the robot is, or any other identifying feature, for example, indicating the functionality of that place. The information can be:

- Geometrical: corresponding to $(x ; y ; \theta)$ coordinates of the robot in relation to the reference system.

- Topological: this localization situates the user in relation to the representative element of the environment. For example: "you are at the Robotics Lab in front of the head's department office".

2) How can I go there? and 3. Where can I go to?: As already said, one of the objectives of a signage system is to determine the actions (indications) needed by the user to move and to facilitate his arrival at his destination. The kind of signal used for this purpose is the Indicator Signal. Those signals are composed by two types of information: a direction and a place. This type of signals are frequently found in our environment, for example, at the airport, we will find a specific boarding gate by following signals such as the one shown in Fig. 1.

\section{A. Information in the signage}

1) Where I am?: In Spain, most of the public buildings use a classification based on zones. In order to facilitate the identification, searching, and the relationship among places, as a general rule, each zone has an alphanumeric id. For example, in our environment, in every building of the campus of the Carlos III University of Madrid (UC3M) there is a map which specifies the location and identification of each zone. Each zone has a specific letter that identifies it. Besides, each building has an identification number, as shown in Fig. 2 . 


\section{$\downarrow \searrow$ Gates $\mathbf{H}^{\mathrm{J}} \boldsymbol{K}$ \\ Puertas M R $\mathbf{D}$}

\section{$\downarrow$ VIP Fast track \\ Acceso VIP}

\section{$\downarrow$ む)}

Fig. 1. Signage at the Barajas Airport (Madrid, Spain)

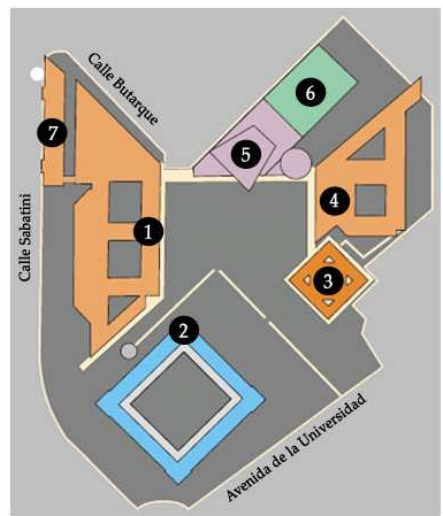

1.- Betancourt Building

2.- Sabatini Building

3.- Rey Pastor Building

4.- Torres Quevedo Building

5.- Padre Soler Building

6.- Sports Complex

7.- Juan Benet Building

Fig. 2. Map of the campus of the Carlos III University of Madrid.

Each building is divided by zones which are identified by letters from A to J. Moreover, every office or room is also identified by an alphanumeric code. Each place of the building uses the scheme shown in Fig. 3.

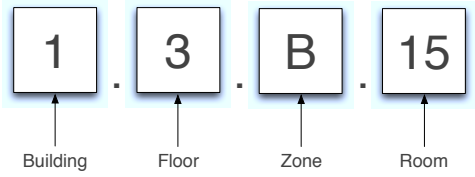

Fig. 3. Alphanumeric code for office identification at the UC3M.

Based on the environment representation, usually found in public buildings, some fundamental concepts related to the signage system are defined:

- Zone: $z_{i}$ is a region or particular area of the environment $E$. Each zone has a name and an alphanumeric identifier.

- Place: $p_{i}$ is the hierarchical representation of the nested zones which contain it. One or several zones form a place.

$$
p_{i}=\left\{z_{1}, \ldots z_{n}\right\}
$$

If several zones form a place then, they are nested, as shown in (2):

$$
z_{1} \subseteq z_{2} \ldots \subseteq z_{n}
$$

For example, when we refer to the "Office 1.3.B.15" at the $\mathrm{UC} 3 \mathrm{M}$, the place will be formed by the following zones:
- $z_{1}=$ Office 1.3.B.15

- $z_{2}=$ Zone B 1.3.B

- $z_{3}=$ Third floor 1.3

- $z_{4}=$ Betancourt Building 1

The most specific zone $z_{1}$ has an id given by an alphanumeric character, which will be called $i d_{1}$. The next zone $z_{n+1}$ has an id with a higher level of abstraction than the one used for $z_{1}$ (see Fig. 4).

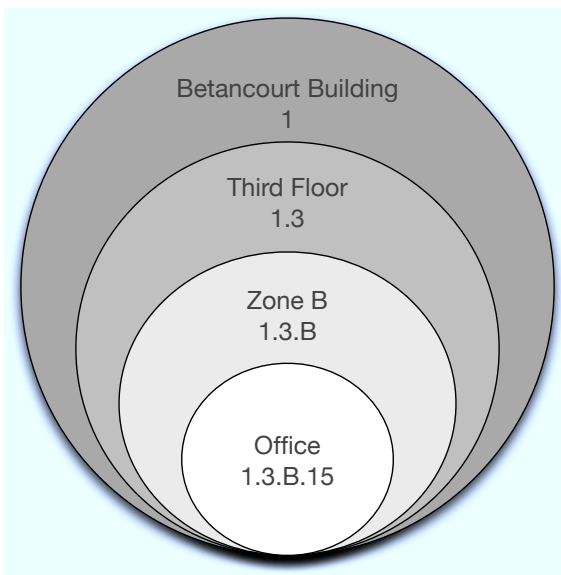

Fig. 4. Representation of the place "Office 1.3.B.15".

2) Where can I go to?, and How can I get there?: Two concepts are defined, for the signage system, that indicate the robot where it can go to, and how to get there:

- Connection: a place can be connected directly or indirectly to another place. A connection is then defined as the possible place or places that the robot can go to from its current place.

A connection is formed by: a place or a list of places, and an action.

- Action: it indicates the action that the robot must execute to get the place indicated by the connection. The action can be:

- Topological: for example: "turn rigth", "move forward", "keep to the left", etc.

- Geometrical: in the case that a geometric map is available, then, the indication may tell the robot to go to a specific coordinate $(x, y, \theta)$.

Finally, a signal $s_{0}$ is formed by a place $p_{0}$, which indicates a current place, and a connection $c_{i}$ or a list of connections $c_{1}, \ldots c_{n}$. A connection $c_{i}$ indicates the place $p_{i}$ where the robot can go to from the place $p_{0}$ indicated the signal by executing an action $a_{i}(i=\{1,2, \ldots, n\})$ (see Fig. 5).

\section{IMPLEMENTATION IN AN AUTONOMOUS ROBOT}

In social robotics, the Human Robot interaction (HRI) is intended to be "easy" since must be oriented to non-expert users, without a technological background. The main applications of social robots are related to domestic assistance, disabled or elderly assistance [18], health care [19], guidance [20], entertainment, among others [21]. Maggie is the social robot developed by the Robotics Lab of the Carlos III University of 


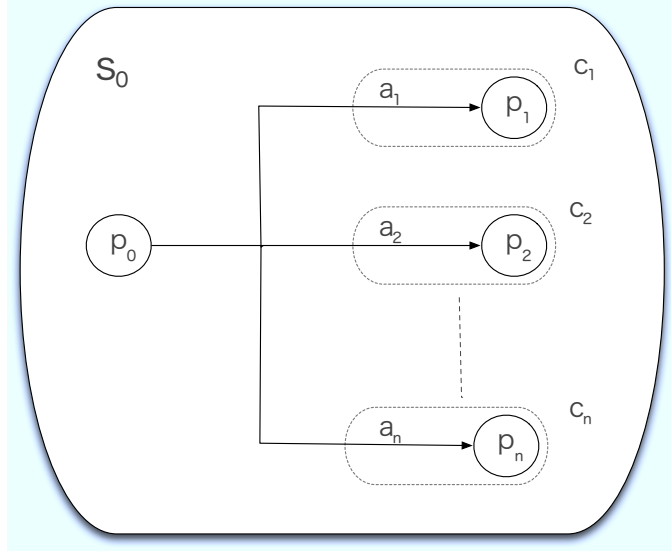

Fig. 5. Signal representation.

Madrid [22]. As shown in Fig. 6, Maggie has been designed in order to have an attractive appearance for the users. The movement of Maggie is possible thanks to a wheeled base.

In previous works, [23] [24], a preliminary design of the signal detection system was shown. In those works high frequency (HD) antennae were selected, in the current work, it has been decided to use UHF RFID readers. The reason is because those readers give a greater reading range $(80 \mathrm{~cm}$ approximately) and it is possible to use tags with no battery (passive tags). Hence, two UHF RFID readers have been placed at both sides of Maggie (see Fig. 6). Due to the features of the antennae, it is possible to situate the RFID tags in the walls of the environment (our department at the university). Therefore, the robot navigates in a safer way without requiring the high precision demanded by the HF system.

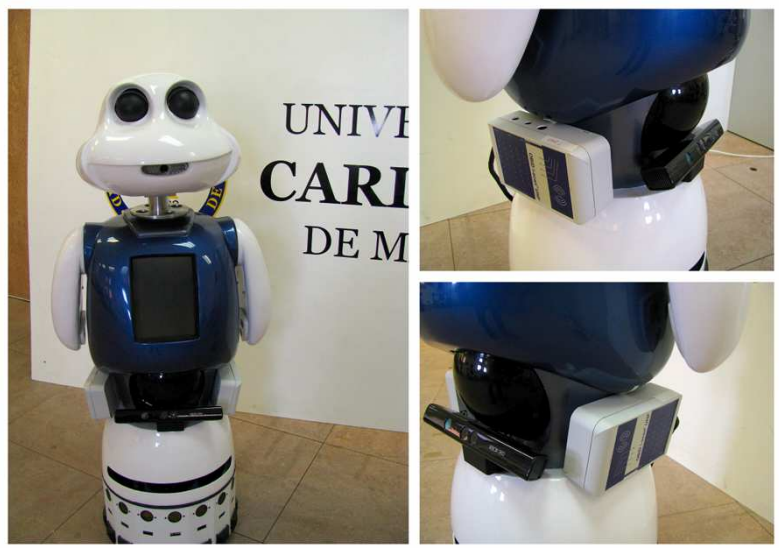

Fig. 6. The robot Maggie with the UHF antennae.

\section{A. RFID tags information}

The RFID tags store the following information:

- An id that represents the type of information stored by the tag: navigation information or useful information for object recognition.

- The signal information: place, connections, and actions.
The basic structure of a signal is modeled using a XML file with the following tags:

1) Place: A place is identified by the element $\langle$ place $\rangle$. This is the main element and contains the different zones that form the place. The definition of a place in XML is made as follows:

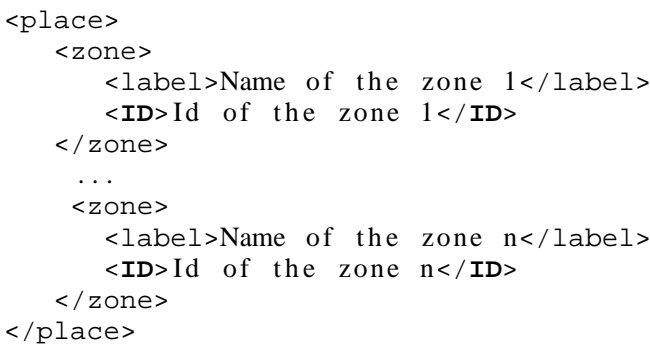

2) Connection: A connection is formed by a place and an action. In XML the following structure is used to define it:

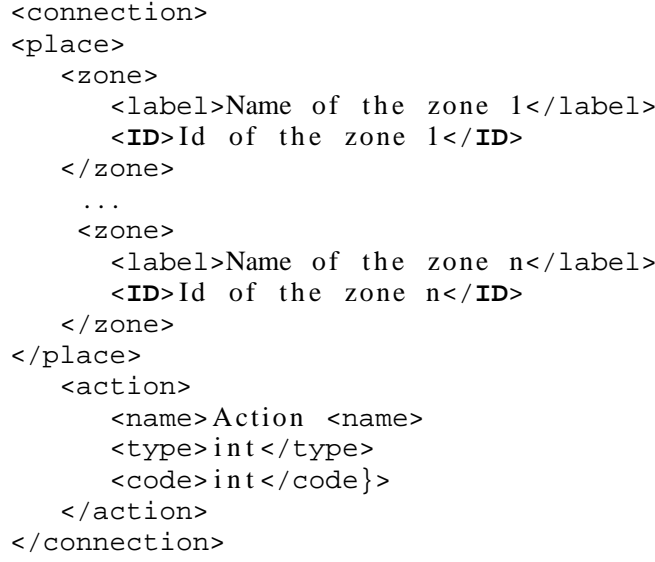

Therefore, a signal is formed by:

- A place that indicates the current place indicated by the signal $(<$ place $>)$.

- A connection, or list of connections, which shows the places that can be reached from the place indicated by the signal, and the actions needed (<connection $>$ ).

\section{NAVIGATION ALgORITHM}

The main objective of the proposed navigation system is that the robot can imitate the human behaviour using the existing signals of the environment. This signals must be used as references during the navigation to arrive successfully at the final destination.

The developed algorithm starts from the following premises:

1) The robot has an assigned goal or destination.

2) The environment is unknown.

3) The robot does not memorize the visited places.

4) In order to reach the goal, the system will only use the information given by the signage system.

The orientation and goal searching process in a robot is basically similar to the one executed by humans in the same situation. As a first step, we search for a sign with the needed information (signal searching). Next, once the information is obtained, we analyze it and we execute the action to arrive at our destination. 
The RFID tags, used as the signals for the navigation system, are situated in the environment and accessible for the robot during the navigation. Since the robot has a predefined destination, then, the robot starts to explore its environment searching for the signals. When one signal is found, the robot reads it and analyzes the obtained data to execute the right actions to reach its goal.

There are different situations or cases:

\section{A. Case 1: the goal coincides with the current place.}

This is the most basic case. The robot finds a signal $\left(s_{i}\right)$, reads it and interprets the information. Then, the robot compares the name and/or the id of the assigned goal $\left(p_{G}\right)$ with the current place indicated by the tag $\left(p_{0}\right)$. In this case, $p_{G}=p_{0} \Rightarrow$ the robot has arrived at its destination, see Fig. 7 .

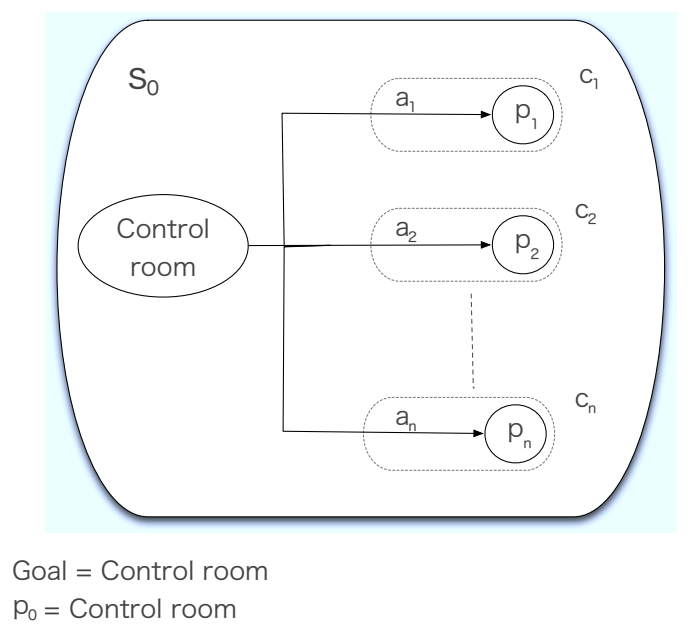

Fig. 7. Case 1: The goal coincides with the current place

\section{B. Case 2: the goal is directly indicated in one connection.}

If $p_{G} \neq p_{0}$ then, the goal place $p_{G}$ must be compared with the place indicated by each connection $c_{i}$ os the signal.

In case of existing a connection that indicates the goal, the robot executes the indicated action and moves toward it.

Example: If the current place is "Office 1.3.B.15" and the connections are given by $\left(\left(p_{c}, i d_{c}\right), a_{c}\right)$ :

- $c_{1}=\{($ Organization Engineering Department,1.3.A $)$, Move to the right $\}$

- $c_{2}=\{($ Robotics Lab I,1.3.C.12), Move to the left $\}$

If the goal is $p_{G}=($ Robotics Lab I, 1.3.C.12), then the robot will execute the action indicated by $c_{2}=$ Move to the left, since $p_{G}=p_{1}$, (Fig. 8).

\section{Case 3: the goal is indirectly indicated in a connection.}

In this case, the signal does not explicitly indicate how to get the goal, so we use the id of each zone to guide the robot to the goal.

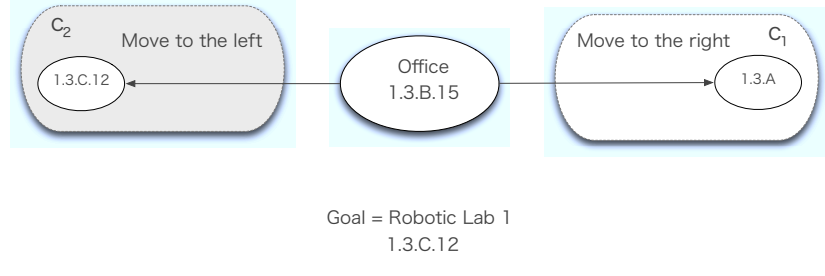

Fig. 8. Case 2: The goal coincides with a connection.

Example: If the current place is "Office 1.3.B.15" and the connections are given by $\left(\left(p_{c}, i d_{c}\right), a_{c}\right)$ :

- $c_{1}=\{($ Organization Engineering Department,1.3.A $)$,

Move to the right $\}$

- $c_{2}=\{($ Robotics Lab I, 1.3.C.12), Move to the left $\}$

If the goal is $p_{G}=$ (Meeting room, 1.3.A.05), when the robot checks that the goal does not coincide with the current place, nor with the connections, then, it will look for a similar id. That is, it will search for a generic id contained in the goal id:

The id 1.3.A.05 is decomposed and the robot now compares each part sequentially, in the following order, until it finds a coincidence:

- 1.3.A.05

- 1.3.A

Then, it compares the zone 1.3.A. If this zone corresponds to one of the connections, then, the robot executes the action indicated by the signal. If not, the id is decomposed again until all the comparisons are made. In the example, the robot finds the connection to zone 1.3.A and executes the indicated action: $c_{1}=$ Move to the right . After that, the robot will find another signal and will repeat the algorithm until it reach the goal, see Fig. 9.

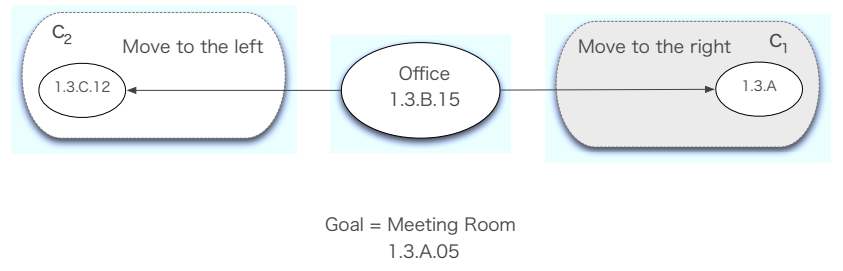

Fig. 9. Case 3: The goal coincides with a zone of one connection

\section{Case 4: The goal is not indicated.}

If none of the previous cases happens, then, the robot can select among the following actions:

- To explore searching for a signal.

- To execute the last action indicated by a signal, in case 2 or 3.

- To wait to be assigned with a new goal, or to receive external information (e.g. through HRI).

In real life generic signals are used to indicate common places such as, the "Exit" signals, the "All directions" found in highways, etc. In our design, connections to generic places are proposed. Therefore, the robot has other options in case 
of being in case 4 so, if it want to go to another floor, it will look for the "Elevators" generic signal.

In summary, the process can be described as follows:

1) The assigned goal is compared with the place indicated by the signal.

2) If there is a coincidence then, this means that the robot has arrived at its destination.

3 ) If the current place does not coincide with the goal place then, the robot look for a connection which indicated how to get to the goal.

4) In case of coincidence, the action (or set of actions), to reach the goal or the next signal, is executed.

5) In case of no coincidence, the robot can select among the actions previously explained in case 4 .

\section{EXPERIMENTAL RESULTS}

This section presents two experiments made to prove the successful performance of the signage system and the navigation algorithm proposed in this paper. These experiments were carried out on the third floor of the Betancourt building of the Carlos III University of Madrid. Fig. 10 shows a view of the navigation area of the robot and its initial location.

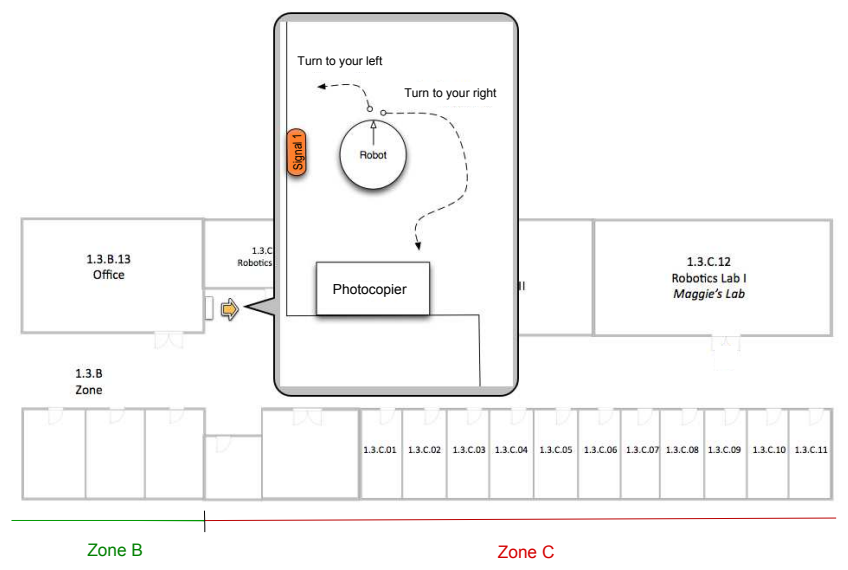

Fig. 10. Third floor map

\section{A. Experiment 1}

\section{Objective}

The robot must go to the Robotics Lab II (1.3.C.13).

\section{Description}

Initially, the robot is beside the photocopier, which is located in the corridor in the zone $\mathrm{C}$. The robot does not know its own location until it reads the signal (RFID tag) also situated next to the photocopier. This experiment tests the behaviour of the robot when a mobile obstacle is situated at the goal's position. Therefore, the robot will not be able to read the signal's goal.

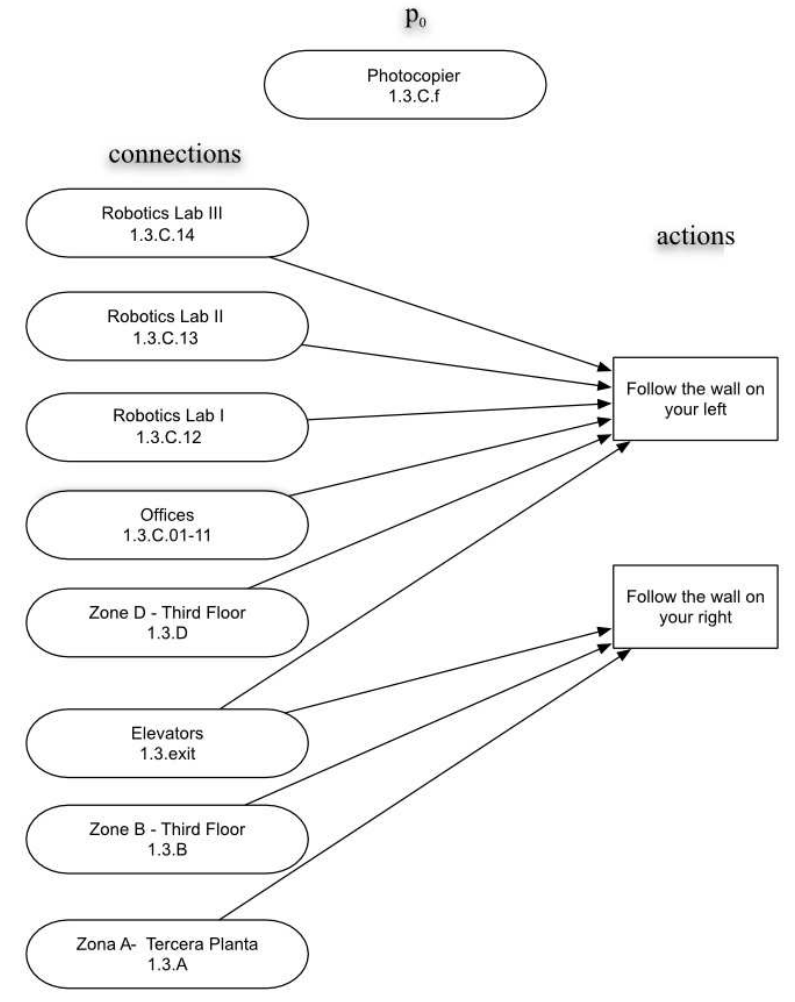

Fig. 11. Information stored in the first signal

Input data

- Assigned goal: Robotics Lab II- 1.3.C.13

- First detected signal: place: photocopier - 1.3.C.f

\section{Process}

Initially, the robot detects the first signal and reads the information about its current location and the possible places where the robot can go to from that place. Fig. 11 shows the information stored in this first signal.

As already explained, the robot compares the assigned goal with the place indicated by the signal. If those places are different, then it starts to compare the goal with the places indicated by the connections. In this occasion, the assigned goal corresponds to one of the connections. Then, the robot activates the "follow the wall on your left" skill and is executed until it finds a new signal.

As shown in Fig. 12, when the robot arrives to its destination, it is not able to read the correspondent signal since there is an object (the grey square) in front of it. Therefore, the robot continues navigating executing the last indicated skill. However, when it finds the next signal (situated at 1.3.C.12), the assigned goal is indicated in one connection. Hence, the robot executes the signaled skill: "follow the wall on your right" and turns round. Now, the obstacle is not longer at the 
corridor, so the robot successfully finds the signal situated at the destination place, see Fig. 13.

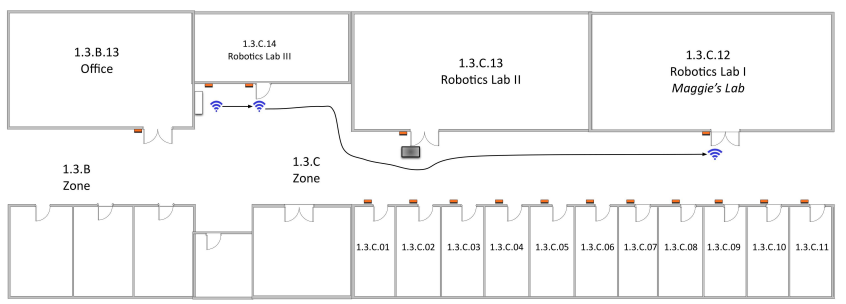

Fig. 12. Experiment 2: Obstacle situated at the destination place

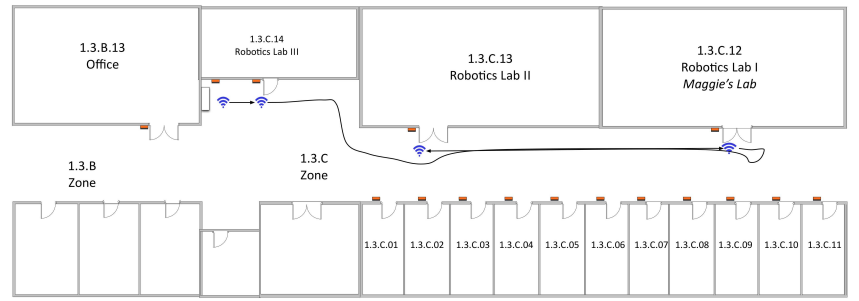

Fig. 13. Experiment 2: Final trajectory of the robot

\section{Results}

Although the robot did not detect the signal at the goal location due to the presence of an obstacle, using the proposed navigation algorithm, the robot finally fulfilled its navigation task: "Go to the Robotics Lab II".

\section{B. Experiment 2}

\section{Objective}

The robot must go to its lab (1.3.C.12) and turn the TV off.

\section{Description}

Again, the robot is initially situated beside the photocopier and it does not know its own location until it reads the signal. The robot must go through the corridor, enter the Robotics Lab I (Maggie's Lab) 1.3.C.12 and turn the TV off.

\section{Input data}

- Assigned goal: TV of the Robotics Lab I- 1.3.C.12.TV

- First detected signal: place: photocopier - 1.3.C.f

\section{Process}

In this experiment the robot task is composed of two objectives:

1) "Go to the TV of the Robotics Lab I".

2) "Turn the TV off".
Objetive 1: "Go to the TV of the Robotics Lab I": As already said, the robot is initially situated next to the photocopier (1.3.C.f). The robot reads the first signal and compare the connections with the assigned goal. As in the previous experiment, Fig. 11 shows the information stored in this first signal.

As can be observed, there is not an exact coincidence with the goal point id "1.3.C.12.TV" (Case 3). Therefore, the system looks for a generic zone that approximates it to the goal. In this case, the most suitable connection is "Robotics Lab I 1.3.C.12".

The robot navigates through the environment using the detected RFID signals. It stops when it find its partial goal: Robotics Lab I- 1.3.C.12. Once the robot arrives at 1.3.C.12 (it detects the correspondent signal), the robot search for a connection which corresponds to the goal point. As shown in Fig. 14, there is a connection which says the robot to "Enter" the lab.

For this experiment, another signal was situated inside the 1.3.C.12 lab, next to its entrance. In Fig. 14, the information stored in this signal is also shown. The added connection specifies the goal place (TV of the Robotics Lab I) and the required action to get there (Go to point $(-0.2,-1.0,-1.7)$ ).

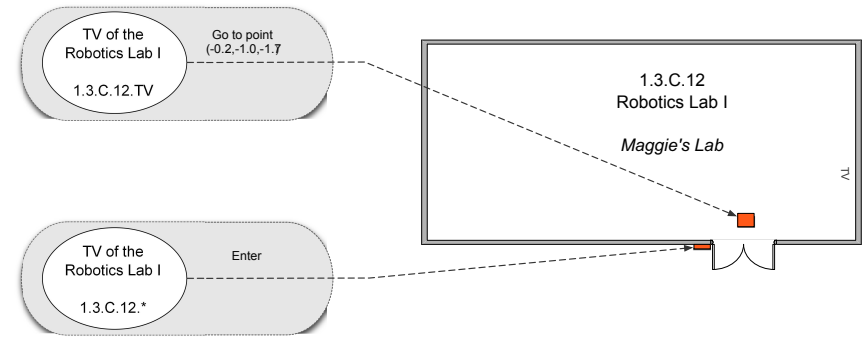

Fig. 14. Robotics Lab I map

In this experiment, apart from using topological actions, the robot is also using a geometric action. The geometric information is used when the topological one is not sufficient, or if the robot is in a non-structured environment. This is the case of our lab since there are tables, chairs, and other objects.

In the XML file of the signal inside the lab, the geometric connection is specified using the following elements:

- <name $>$ : specifies the action's name. In this case GTP $(-0.2,-1.0,-1.7)$ indicates that the robot will activate the skill "Go to Point", where the coordinate is: $x=-0.2, y=-1.0$ y $\theta=-1.7$, expressed in meters and radians respectively.

- <GeometricMap>: In order to activate the "Go to Point" skill, the system requires a geometric map of the environment. This map has been previously made and stored in the URL indicated by <direccion>.

- <localization>: this field indicates the geometric localization of the robot. This localization is in relation to the geometric map when the signal is detected. Therefore, the robot localize itself and executes the "Go to Point" skill.

Objective 2: "Turn the TV off": Once the robot is in front of the TV, it activates its remote control system based on IR 
[25]. This skill enables the IR receiver/emitter integrated in Maggie in order to control different electronic devices of its environment. Then, the system executes the command: "Turn the TV off" when the robot arrives at its final destination.

\section{Results}

As shown, the robot was able to successfully arrive at its assigned destination. It moved around the environment according to the instructions given by the detected RFID signals. Finally, in Fig. 15 the complete route followed by the robot is shown.

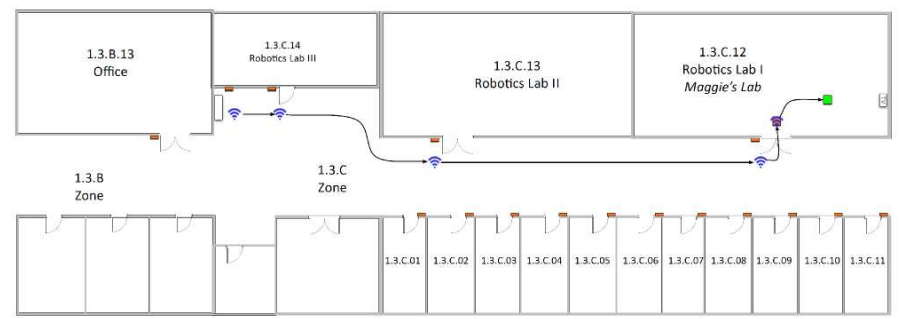

Fig. 15. The followed route to get the final destination

\section{CONCLUSIONS AND FUTURE WORKS}

In this paper, a signage system to support the navigation of autonomous robots is presented. This system is intended to be used in indoor and structured environments such as homes, public buildings, etc. The used signals are low cost RFID tags which do not disturb the navigation of people, nor make a negative impact in the environment.

In this work, unlike other related works, the designed system is able to complement topological with geometric information. Moreover, it does not required previous topological maps. The robot can use the signals to guide itself and, in case of getting lost or not reading a signal, the robot can keep navigating using other signals to orientate itself again.

The proposed signage system has been successfully implemented in a real environment. It has been proved that signals are a great support for autonomous robot navigation, when they do not have a previous knowledge of the environment. In relation to the response time, during the reading process, the average value is around $25 \mathrm{~ms}$, so it can be considered as an acceptable delay time. This work represents a base for possible applications in "smart homes". This technology could be applied not only for improving the people's qualify of life, but also, for facilitating the navigation of the robots which assist them at home.

Finally, as future works, the authors's intention is to try the performance of the proposed signage system and the developed navigation algorithm in other environments, such as hospitals, museums, shopping centers, etc.

During real experiences, the robot may not detect the RFID tags (e.g. due to the lack of signals in a certain area) and it get lost or it may has a cyclic behaviour. In that case, this system must be completed using other navigation system or another kind of signals, such as the existing visual signals of the environment. Other solutions could be the use of learning techniques to learn the already visited places and paths, finally, the robot could ask for help to the people around.

Moreover, since the signage system is intended for social robots, it would be quite interesting to include a signal-writing method based on HRI. Therefore, the social robot will be able to write and modify the content of the signals following the instructions given by a person.

\section{ACKNOWLEDGMENT}

The authors gratefully acknowledge the funds provided by the Spanish Government through the project called "A new approach to social robotics" (AROS), of MICINN (Ministry of Science and Innovation) and through the RoboCity2030II-CM project (S2009/DPI-1559), funded by Programas de Actividades I+D en la Comunidad de Madrid and cofunded by Structural Funds of the EU.

\section{REFERENCES}

[1] E. C. Tolman, "Cognitive maps in rats and men," Psychological Review, vol. 55, pp. 189-208, 1948.

[2] R. Golledge, Wayfinding Behavior. Cognitive mapping and other spatial processes. The Johns Hopkins University Press, 1999.

[3] K. V. Istomin and M. J. Dwyer, "Finding the Way: A Critical Discussion of Anthropological Theories of Human Spatial Orientation with Reference to Reindeer Herders of Northeastern Europe and Western Siberia," Current Anthropology, vol. 50, no. 1, pp. 29-49, February 2009. [Online]. Available: http://dx.doi.org/10.1086/595624

[4] K. Lynch, The Image of the City. Cambridge MA: MIT Press, 1960.

[5] L. C. D. Silva, C. Morikawa, and I. M. Petra, "State of the art of smart homes," Engineering Applications of Artificial Intelligence, no. 0, pp. -, 2012. [Online]. Available: http://www.sciencedirect.com/science/ article/pii/S095219761200098X

[6] M. Mata, J. Armingol, A. de la Escalera, and M. Salichs, "A visual landmark recognition system for topological navigation of mobile robots," in Robotics and Automation, 2001. Proceedings 2001 ICRA. IEEE International Conference on, vol. 2, 2001, pp. 1124 - 1129 vol.2.

[7] F. Wen, K. Yuan, W. Zou, X. Chai, and R. Zheng, "Visual navigation of an indoor mobile robot based on a novel artificial landmark system," in Mechatronics and Automation, 2009. ICMA 2009. International Conference on, 2009, pp. $3775-3780$.

[8] M. Darianian and M. Michael, "Smart home mobile rfid based internet of things systems and services," in Advanced Computer Theory and Engineering, 2008. ICACTE '08. International Conference on, dec. 2008, pp. $116-120$.

[9] D. Ding, R. A. Cooper, P. F. Pasquina, and L. Fici-Pasquina, "Sensor technology for smart homes," Maturitas, vol. 69, no. 2, pp. 131 - 136, 2011. [Online]. Available: http://www.sciencedirect.com/science/article/ pii/S0378512211000983

[10] L. Caviglione, M. Coccoli, and A. Grosso, "A framework for the delivery of contents in rfid-driven smart environments," in RFID-Technologies and Applications (RFID-TA), 2011 IEEE International Conference on, sept. 2011, pp. $45-49$.

[11] H.-H. Hsu, C.-N. Lee, and Y.-F. Chen, "An rfid-based reminder system for smart home," in Advanced Information Networking and Applications (AINA), 2011 IEEE International Conference on, march 2011, pp. 264 -269 .

[12] S. Park and S. Hashimoto, "Autonomous mobile robot navigation using passive rfid in indoor environment," Industrial Electronics, IEEE Transactions on, vol. 56, no. 7, pp. 2366 -2373, july 2009.

[13] B.-S. Choi, J.-W. Lee, J.-J. Lee, and K.-T. Park, "A hierarchical algorithm for indoor mobile robot localization using rfid sensor fusion," Industrial Electronics, IEEE Transactions on, vol. 58, no. 6, pp. 2226 -2235, june 2011.

[14] E. Di Giampaolo and F. Martinelli, "Robot localization by sparse and passive rfid tags," in Industrial Electronics (ISIE), 2010 IEEE International Symposium on, july 2010, pp. $1937-1942$.

[15] V. Kulyukin, C. Gharpure, J. Nicholson, and G. Osborne, "Robotassisted wayfinding for the visually impaired in structured indoor environments," Auton. Robots, vol. 21, no. 1, pp. 29-41, 2006. 
[16] V. Kulyukin, C. Gharpure, and J. Nicholson, "Robocart: toward robotassisted navigation of grocery stores by the visually impaired," in Intelligent Robots and Systems, 2005. (IROS 2005), 2005, pp. $2845-$ 2850.

[17] V. Kulyukin, A. Kutiyanawala, E. LoPresti, J. Matthews, and R. Simpson, "iwalker: Toward a rollator-mounted wayfinding system for the elderly," in RFID, 2008 IEEE International Conference on, 2008, pp. $303-311$.

[18] K. Wada, T. Shibata, T. Musha, and S. Kimura, "Robot therapy for elders affected by dementia," Engineering in Medicine and Biology Magazine, IEEE, vol. 27 , no. 4 , pp. $53-60$, july aug. 2008.

[19] C. Breazeal, "Social robots for health applications," in Engineering in Medicine and Biology Society,EMBC, 2011 Annual International Conference of the IEEE, 30 2011-sept. 3 2011, pp. 5368 -5371.

[20] M. Shiomi, T. Kanda, D. Glas, S. Satake, H. Ishiguro, and N. Hagita, "Field trial of networked social robots in a shopping mall," in Intelligent Robots and Systems, 2009. IROS 2009. IEEE/RSJ International Conference on, oct. 2009, pp. $2846-2853$.

[21] F. Hegel, M. Lohse, A. Swadzba, S. Wachsmuth, K. Rohlfing, and B. Wrede, "Classes of applications for social robots: A user study," in Robot and Human interactive Communication, 2007. RO-MAN 2007. The 16th IEEE International Symposium on, aug. 2007, pp. 938 -943.

[22] M. A. Salichs, R.Barber, A.M.Khamis, M.Malfaz, J.F.Gorostiza, R.Pacheco, R.Rivas, A.Corrales, and E.Delgado, "Maggie: A robotic platform for human-robot social interaction," in IEEE International Conference on Robotics, Automation and Mechatronics, IEEE, Ed., Bangkok. Thailand, June 2006.

[23] A. Corrales and M. A. Salichs, "Integration of a rfid system in a socia robot," in Progress in Robotics, ser. Communications in Computer and Information Science, J.-H. Kim, S. S. Ge, P. Vadakkepat, N. Jesse, A. Al Manum, S. Puthusserypady K, U. Rckert, J. Sitte, U. Witkowski, R. Nakatsu, T. Braunl, J. Baltes, J. Anderson, C.-C. Wong, I. Verner, and D. Ahlgren, Eds. Springer Berlin Heidelberg, 2009, vol. 44, pp. 63-73.

[24] A. Corrales and M. Salichs, "Use of rfid technology on a mobile robot for topological navigation tasks," in RFID-Technologies and Applications (RFID-TA), 2011 IEEE International Conference on, sept. 2011, pp. $408-414$.

[25] J. Salichs, A. Castro-Gonzalez, and M. A. Salichs, "Infrared remote control with a social robot," in Progress in Robotics, ser. Communications in Computer and Information Science, J.-H. Kim, S. S. Ge, P. Vadakkepat, N. Jesse, A. Al Manum, S. Puthusserypady K, U. Rckert, J. Sitte, U. Witkowski, R. Nakatsu, T. Braunl, J. Baltes, J. Anderson, C.C. Wong, I. Verner, and D. Ahlgren, Eds. Springer Berlin Heidelberg, 2009, vol. 44, pp. 86-95.

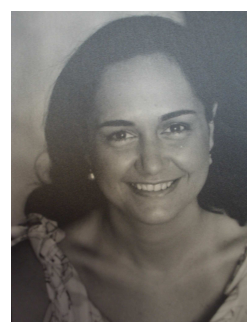

Maria Malfaz received the B.Sc. degree in physics science from La Laguna University, La Laguna, Spain, in 1999, and the M.Sc. degree in control systems from the Imperial College of London, London, U.K., in 2001. She received the Ph.D. degree in industrial engineering from the University Carlos III of Madrid, Madrid, Spain, in 2007, with the topic "Decision Making System for Autonomous Social Agents Based on Emotions and Self-learning." She is currently an Assistant Professor in the Department of System Engineering and Automation of the University Carlos III of Madrid, Madrid, Spain. Her current research area follows the line carried out in her thesis.

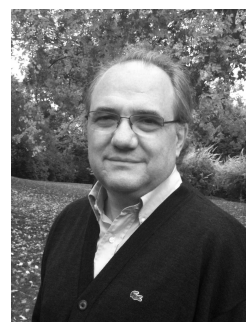

Miguel A. Salichs received the electrical engineering and $\mathrm{Ph} . \mathrm{D}$. degrees from Polytechnic University of Madrid, Madrid, Spain, in 1978 and 1982, respectively. He is currently a Full Professor of the Systems Engineering and Automation Department at the Carlos III University, Madrid, Spain. His research interests include autonomous social robots, multimodal human-robot interaction, mind models, and cognitive architectures. Dr. Salichs was member of the Policy Committee of the International Federation of Automatic Control (IFAC), chairman of the Technical Committee on Intelligent Autonomous Vehicles of IFAC, the responsible of the Spanish National Research Program on Industrial Design and Production, the President of the Spanish Society on Automation and Control (CEA) and the Spanish representative at the European Robotics Research Network (EURON).

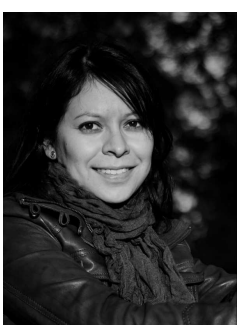

Ana Corrales received in 2004 the B.Sc. degree in Systems Engineering from Los Andes University, Mérida,Venezuela. In 2008, she received the M.Sc. degree in Robotics and Automation from the Carlos III University of Madrid, Madrid, Spain, where she also received, in 2012, the Ph.D. degree in Industrial Automation Engineering, with the topic "Navigation of Social Robots using signaling." Dr. Corrales got a scholarship in 2004 to postgraduate studies during 3 years and later, she was Assistant Teacher in the Department of Systems Engineering and Automation of the Carlos III University of Madrid from 2007 to 2012. She has worked in several research projects related to social robots, autonomous navigation in mobile robots and RFID technologies. Currently she is a collaborator member at the Robotics Lab of Carlos III University of Madrid. 